\title{
Corrosion inhibition capacity of two heterocyclic oximes on copper in nitric acid: electrochemical, quantum chemical and surface morphological investigations
}

\author{
C. Sini Varghese ${ }^{a}$, K. Joby Thomas ${ }^{a^{*}}$, Vinod P. Raphael ${ }^{b}$ and K. S. Shaju ${ }^{b}$
}

${ }^{a}$ Research Division, Department of Chemistry, St. Thomas' College (Autonomous) Thrissur, Kerala, 680001,India ${ }^{b}$ Department of Chemistry, Government Engineering College, Thrissur, Kerala, 680009, India

\begin{tabular}{l}
\hline C H R O N I C L E \\
\hline Article history: \\
Received September 9, 2018 \\
Received in revised form \\
November 9, 2018 \\
Accepted December 14, 2018 \\
Available online \\
December 14, 2018 \\
\hline Keywords: \\
Corrosion \\
Inhibitor \\
Polarization \\
Impedance \\
Adsorption
\end{tabular}

\begin{abstract}
A B S T R A C T
Two heterocyclic oximes (E)-N-hydroxy-1-(pyridin-2-yl)methanimine (Hp2ylm) and (E)-Nhydroxy-1-(pyridin-3-yl)methanimine (Hp3ylm) were synthesized from pyridine-2carbaldehyde and pyridine-3-carbaldehyde, respectively. These oximes were characterized by various spectroscopic tools like UV, IR, MASS and NMR. The inhibition capacity of these oximes against copper corrosion in $0.1 \mathrm{M} \mathrm{HNO}_{3}$ was determined by polarization and impedance spectroscopic studies (EIS). At all concentrations, Hp3ylm exhibited higher inhibition efficiency than Hp2ylm. Attempt was made to illustrate the mechanism of corrosion inhibition by these oximes with the help of adsorption isotherm, scanning electron microscopic (SEM) and quantum chemical studies.
\end{abstract}

\section{Introduction}

Copper is a noble and widely using engineering metal in various fields like electrical, electronics and automobile industries. ${ }^{1}$ Due to the less corrosive behaviour, only powerful oxidizing agents can or react with copper. For the fabrication of electronic components copper has to be dissolved in aggressive solutions and to be electroplated. Some electronic components like printed circuit boards have to be cleaned with corrosive solutions during fabrication. Nitric acid is the aggressive solution frequently used for this purpose in various industries. ${ }^{2}$ During this surface cleaning process it is customary to add corrosion inhibiting agents to combat against corrosion. Scientists are ever in search of novel, efficient and economic corrosion inhibiting species to minimize corrosion. ${ }^{3,4}$ It was proved by the earlier researchers that organic molecules bearing hetero atoms like $\mathrm{N}, \mathrm{O}, \mathrm{S}$ etc and containing aromatic rings act as good corrosion protecting molecules. Many of such molecules are unstable in acidic solutions and possess less solubility. ${ }^{5-12}$

Main objectives of the present investigation are that to prepare new organic molecules having extra stability and good solubility in nitric acid medium, to characterize these molecules using spectral studies, and to determine their corrosion inhibition efficiency on copper surface.

* Corresponding author. Tel.: +9847177695

E-mail address: driobythomask@gmail.com (K. J. Thomas)

(C) 2019 by the authors; licensee Growing Science, Canada

doi: $10.5267 /$ j.ccl.2018.012.001 


\section{Results and Discussion}

\subsection{EIS studies}

Interaction of oximes Hp2ylm and Hp3ylm on the copper surface in $0.1 \mathrm{M}$ nitric acid solution at $30^{\circ} \mathrm{C}$ was determined by impedance studies. By subtracting the solution resistance from the total resistance, charge transfer resistance of copper specimen $\left(\mathrm{R}_{\mathrm{ct}}\right)$ was calculated from the impedance plots. ${ }^{13,14}$ Using equation 1 , double layer capacitance $\left(C_{d l}\right)$ was determined. In this equation $f_{\max }$ represents the maximal frequency and $\mathrm{R}_{\mathrm{ct}}$ is the charge transfer resistance.

$$
C_{d l}=\frac{1}{2 \pi f_{\max } R_{c t}}
$$

Fig. 1(a) and Fig. 1(b) represent the Nyquist plots for $\mathrm{Cu}$ specimens in decimolar nitric acid solution with different amounts of oximes Hp2ylm and Hp3ylm. Due to the roughness of the surface and mass transfer resistance Nyquist plots was not perfect semi circles. ${ }^{15,16}$

The simple equivalent circuit (Randles circuit) consisting of solution resistance $\left(\mathrm{R}_{\mathrm{s}}\right)$ connected in series with a parallel combination of $\mathrm{R}_{\mathrm{ct}}$ and $\mathrm{C}_{\mathrm{dl}}$ as represented in Fig. 2 fitted well on the Nyquist plots.

Impedance response of copper specimens was changed appreciably in the presence of oximes Hp2ylm and Hp3ylm in nitric acid medium. As the concentration of oximes increased, the double layer capacitance values decreased considerably. This can be attributed to the replacement of previously adsorbed water molecules and ions by the organic molecules. ${ }^{17,18}$ The layer of adsorbed molecules creates mass transfer resistance on the copper surface. On analyzing the impedance plots, it is evident that the $\mathrm{R}_{\mathrm{ct}}$ values increased with increase in concentration of added oximes to the aggressive medium. Impedance data and percentage of corrosion inhibition efficiency of Hp2ylm and Hp3ylm are given in Table1.

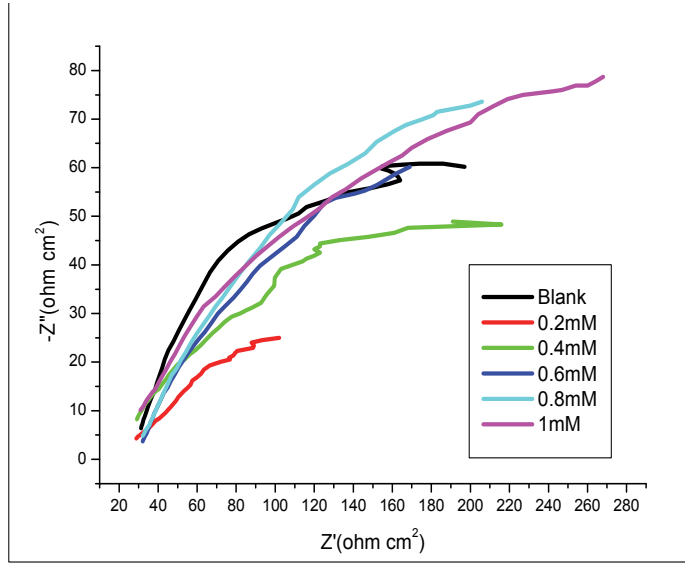

(a)

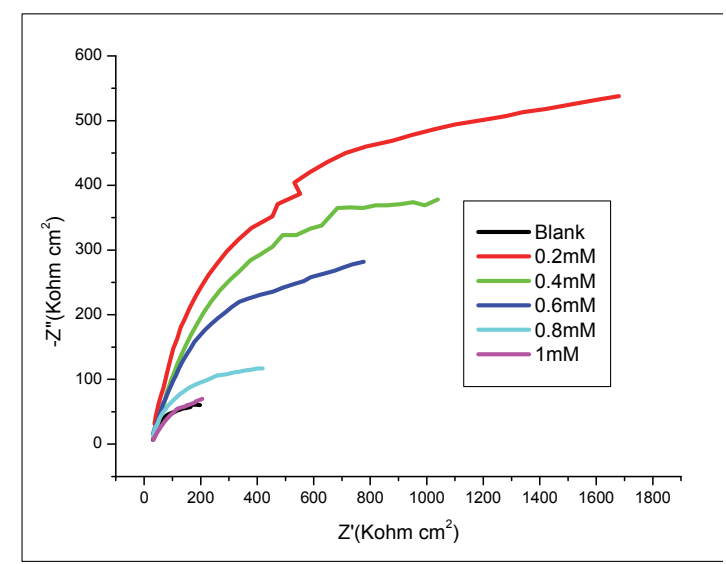

(b)

Fig. 1. Nyquist plots for copper in the absence and presence of a) Hp2ylm and b) Hp3ylm in $0.1 \mathrm{M}$ nitric acid 


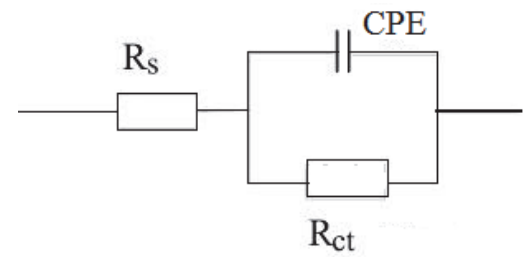

Fig. 2. Equivalent circuit used for the analysis of Nyquist plots

On analysing Table 1, it is unambiguous that the corrosion protection capacity of Hp3ylm is very much higher than that of Hp2ylm at all concentrations. At $1.0 \mathrm{mM}$ concentration, Hp3ylm and Hp2ylm displayed $90.1 \%$ and $26.9 \%$ inhibition efficiency on copper surface, respectively. Even though two oximes are structural isomers, $\mathrm{Hp} 3 y$ lm showed enhanced interaction on $\mathrm{Cu}$ surface in $\mathrm{HNO}_{3}$. When we examine the structural features of Hp3ylm and Hp2ylm, it is understandable that Hp2ylm molecules have the strong tendency to form intra molecular hydrogen bond as depicted in Fig. 3(a). Since there is no possibility of intra molecular hydrogen bonding in Hp3ylm, these molecules mainly associate through inter molecular hydrogen bonds. Large number of Hp3ylm molecules can link together and can make a passive layer on the copper surface. This makes the interaction of Hp3ylm on the copper surface very high than Hp2ylm.

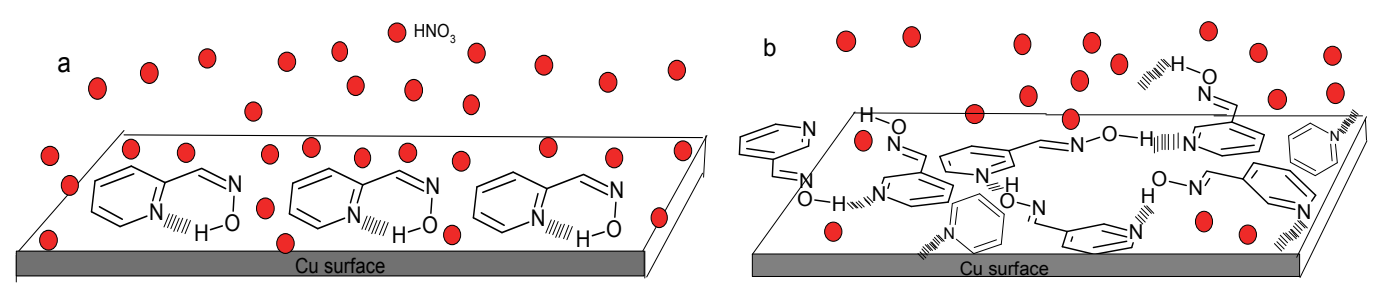

Fig. 3. Interaction of a) Hp2ylm and b) Hp3ylm on copper in $0.1 \mathrm{M} \mathrm{HNO}_{3}$

Table 1. EIS data of $\mathrm{Cu}$ in the absence and presence of Hp2ylm and $\mathrm{Hp} 3 y \mathrm{~lm}$ in $0.1 \mathrm{M} \mathrm{HNO}_{3}$

\begin{tabular}{lllccccc}
\hline Oximes & Parameter & Blank & $0.2 \mathrm{mM}$ & $0.4 \mathrm{mM}$ & $0.6 \mathrm{mM}$ & $0.8 \mathrm{mM}$ & $1.0 \mathrm{mM}$ \\
\hline \multirow{3}{*}{ Hp2ylm } & $\mathrm{C}_{\mathrm{dl}}\left(\mu \mathrm{F} \mathrm{cm}^{-2}\right)$ & 745 & 3716 & 1545 & 2095 & 1439 & 535 \\
& $\mathrm{R}_{\mathrm{ct}}\left(\Omega \mathrm{cm}^{2}\right)$ & 135.90 & 55 & 114 & 121 & 155 & 186 \\
& $\eta_{\mathrm{EIS}} \%$ & - & -147.09 & -19.21 & -12.31 & 12.32 & 26.93 \\
\hline \multirow{3}{*}{ Hp3ylm } & $\mathrm{C}_{\mathrm{dl}}\left(\mu \mathrm{F} \mathrm{cm}^{-2}\right)$ & 745 & 2018 & 334 & 385 & 235 & 157 \\
& $\mathrm{R}_{\mathrm{ct}}\left(\Omega \mathrm{cm}^{2}\right)$ & 135.90 & 294 & 307 & 672 & 879 & 1379 \\
& $\eta_{\text {EIS }} \%$ & - & 53.78 & 55.73 & 79.78 & 84.54 & 90.14 \\
\hline
\end{tabular}

\subsection{Adsorption Studies}

Adsorption isotherms are used to investigate the nature of interaction of molecules on metal surface. ${ }^{19-21}$ Out of the various models of isotherms tried the best one was chosen using correlation coefficient (Table 2). For Hp3ylm, the most fitting isotherm on copper surface was Langmuir adsorption isotherm (Eq. (2)). The graphical representation of the isotherm is given in Fig. 4. The heterocyclic oximes Hp2ylm was having very weak interaction on surface the metal. At lower concentrations, Hp2ylm showed negative corrosion inhibition efficiency, where corrosion rate is accelerated. Thus, we can observe increased dissolution of copper metal when it is treated with $0.1 \mathrm{M}$ $\mathrm{HNO}_{3}$ containing low concentrations of Hp2ylm. Thus, adsorption isotherm could not fit for the interaction of Hp2ylm on copper surface.

Langmuir adsorption isotherm

$$
\frac{C}{\theta}=\frac{1}{K_{a d s}}+C
$$


In this equation $\mathrm{C}, \theta$ and $\mathrm{K}_{\mathrm{ads}}$ represent concentration of inhibiting species, fractional surface coverage, and adsorption equilibrium constant, respectively. ${ }^{21}$

Table 2. Adsorption isotherms and $\mathrm{R}^{2}$ values for $\mathrm{Hp} 3 y \mathrm{~lm}$ on $\mathrm{Cu}$ in $0.1 \mathrm{M} \mathrm{HNO}_{3}$

\begin{tabular}{lc}
\hline Adsorption isotherm & Regression coefficient Hp3ylm \\
\hline Langmuir & 0.945 \\
Freundlich & 0.904 \\
Frumkin & 0.870 \\
Temkin & 0.625 \\
El-Awady & 0.868 \\
Flory-Huggin & 0.705 \\
\hline
\end{tabular}

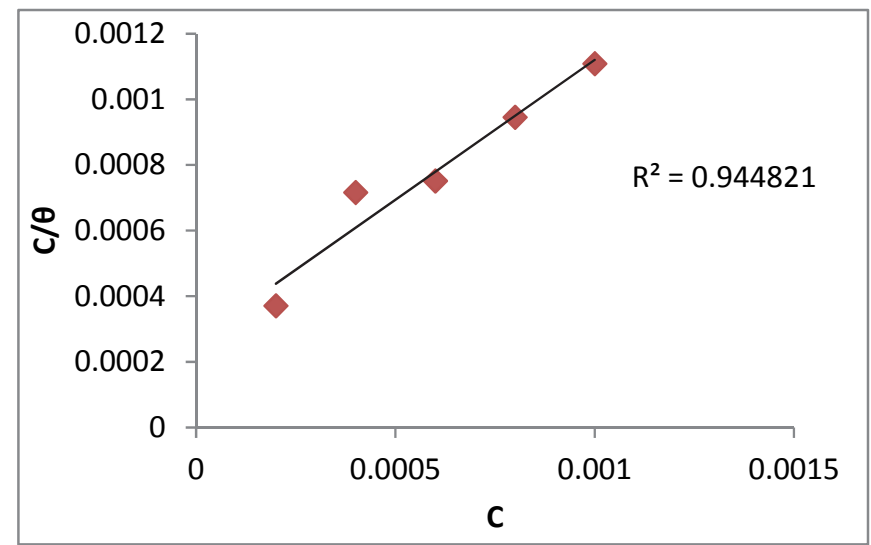

Fig. 4. Langmuir isotherm for $\mathrm{Hp} 3 y l m$ on $\mathrm{Cu}$ in $0.1 \mathrm{M} \mathrm{HNO}_{3}$

The following thermodynamic equation relates $\mathrm{K}_{\mathrm{ads}}$ and standard free energy of adsorption $\left(\Delta \mathrm{G}^{\circ}{ }_{\mathrm{ads}}\right)$ : $\Delta \mathrm{G}_{\mathrm{ads}}^{0}=-\mathrm{RT} \ln \left(55.5 \mathrm{~K}_{\mathrm{ads}}\right)$

In this equation 55.5 is the molar concentration of water, $\mathrm{R}$ and $\mathrm{T}$ denote universal gas constant, and temperature in Kelvin scale respectively. ${ }^{19}$ Generally, the values of $\Delta G^{0}$ ads up to $-20 \mathrm{~kJ} / \mathrm{mol}$ is an indication of electrostatic interaction (physisorption) of inhibitor molecules and the metal, while those around $-40 \mathrm{~kJ} / \mathrm{mol}$ stands for chemisorption. The $\Delta \mathrm{G}^{0}$ ads values lie between -20 and $-40 \mathrm{~kJ} / \mathrm{mol}$, both physisorption and chemisorption was involved during the interaction of molecules on the metal surface. Hp3ylm showed values 3745 and $-30.64 \mathrm{~kJ} / \mathrm{mol}$ for $\mathrm{K}_{\mathrm{ads}}$ and $\Delta \mathrm{G}_{\text {ads }}^{0}$ respectively. These values indicate the strong interaction of Hp3ylm molecules on metal surface in nitric acid medium. The magnitudes of free energy of adsorptions indicate that Hp3ylm molecules adsorb on the metal surface through both physical and chemical forces.

\subsection{Potentiodynamic Studies}

Due to the strong oxidizing power of nitric acid solution, copper will be converted to $\mathrm{Cu}^{2+}$ ions and no protective oxide will remain on the metal surface. This fact is very much visible from the Pourbaix diagram of copper-water system. ${ }^{22,23}$ The reaction between $\mathrm{Cu}$ and nitric acid is explained below:

Anodic dissolution of copper occurs as represented in equation 4. The surface metal atoms convert into ions and diffuse into the bulk of solution.

$\mathrm{Cu} \rightarrow \mathrm{Cu}^{2+}+2 \mathrm{e}$ 
At cathode, the main reactions involving are the reduction of the nitrate ion into nitrous acid or nitric oxide, as per equations 5 and 6 . If the solution is aerated, there is a chance for the reduction of dissolved oxygen on copper surface ${ }^{24}$ in accordance with Eq. 7.

$$
\begin{aligned}
& \mathrm{NO}_{3}{ }^{-}+3 \mathrm{H}^{+}+2 \mathrm{e} \rightarrow \mathrm{HNO}_{2}+\mathrm{H}_{2} \mathrm{O} \\
& \mathrm{NO}_{3}{ }^{-}+4 \mathrm{H}^{+}+3 \mathrm{e} \rightarrow \mathrm{NO}+2 \mathrm{H}_{2} \mathrm{O} \\
& \mathrm{O}_{2}+4 \mathrm{H}^{+}+4 \mathrm{e} \rightarrow 2 \mathrm{H}_{2} \mathrm{O}
\end{aligned}
$$

Tafel plots of copper in $0.1 \mathrm{M}$ nitric acid in the presence and absence of the Hp2ylm and Hp3ylm were analyzed to obtain the data. Polarization parameters such as corrosion current density ( $\mathrm{I}_{\text {corr }}$ ), corrosion potential $\left(\mathrm{E}_{\mathrm{corr}}\right)$, anodic Tafel slopes $\left(\mathrm{b}_{\mathrm{a}}\right)$, cathodic Tafel slopes $\left(\mathrm{b}_{\mathrm{c}}\right)$ and inhibition efficiency $\eta_{\mathrm{pol}} \%$ are represented in Table 3. It is clear from the table that as the concentration of the heterocyclic oximes increases corrosion current density of copper specimens ( $\mathrm{I}_{\text {corr }}$ ) lowered appreciably. The corrosion inhibition efficiency was increased with the concentration of oximes. Similar to the results of EIS studies, Hp3ylm displayed enhanced inhibition efficiency than Hp2ylm at all concentrations.

On close examination of the Tafel plots of Hp2ylm, Fig. 5(a) and polarisation parameters, it may be concluded that the corrosion potentials of copper at various concentrations of Hp2ylm changes to cathodic and anodic directions and the inhibitive response of this oxime can be regarded as mixed type i.e., Hp2ylm affects both sites of corrosion (anodic and cathodic) more or less uniformly. Also the cathodic and anodic slopes of the Tafel curve changes in each scans, which can be taken as an additional supporting evidence for the above argument.

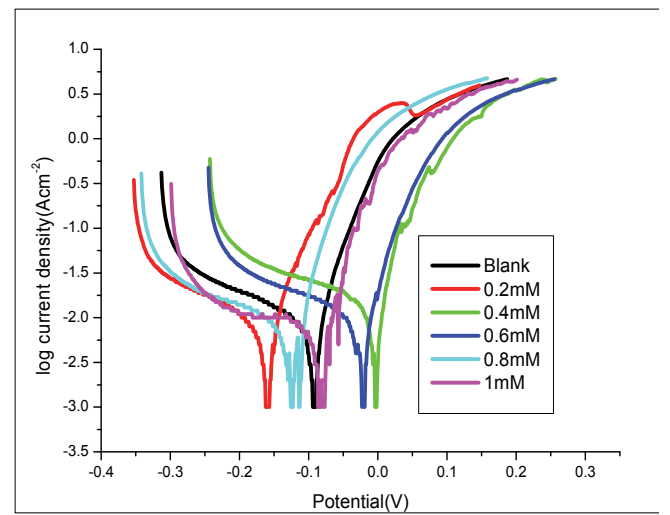

(a)

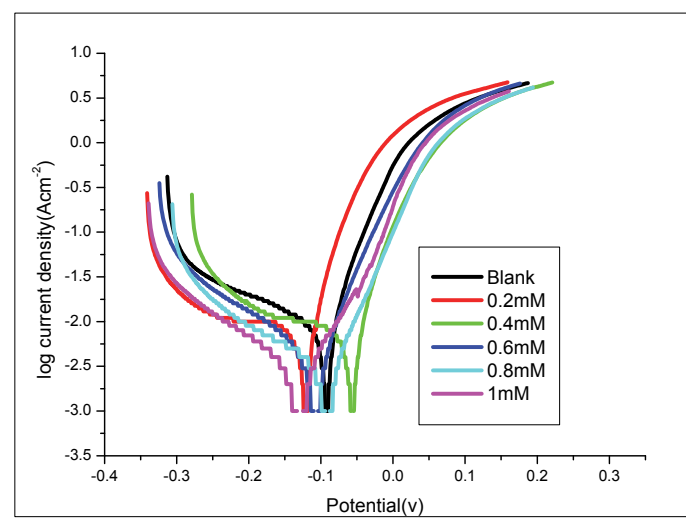

(b)

Fig. 5. Tafel plots for copper in the absence and presence of a) Hp2ylm and b) Hp3ylm in $0.1 \mathrm{M}$ nitric acid

On evaluating the polarization curves of copper in the presence and absence of Hp2ylm and Hp3ylm (Fig. 5), one can see the change of cathodic and cathodic slopes as compared to that of blank, suggesting that these molecules inhibit the cathodic and anodic process of corrosion appreciably, in other words these oximes are having the behaviour of mixed type corrosion inhibitor. The corrosion inhibition efficiencies of Hp3ylm on the copper metal surface were higher at all concentrations than that of Hp2ylm. These results are in good agreement with the results of impedance analysis. 
Table 3. Tafel parameters of $\mathrm{Cu}$ in the presence and absence of oximes in $0.1 \mathrm{M} \mathrm{HNO}_{3}$

\begin{tabular}{clcccccc}
\hline Oxime & Parameter & Blank & $0.2 \mathrm{mM}$ & $0.4 \mathrm{mM}$ & $0.6 \mathrm{mM}$ & $0.8 \mathrm{mM}$ & $1.0 \mathrm{mM}$ \\
\hline \multirow{3}{*}{ Hp2ylm } & $-\mathrm{E}_{\text {corr }}(\mathrm{mV} / \mathrm{SCE})$ & 240 & 339 & 100 & 158 & 274 & 271 \\
& $\mathrm{I}_{\text {corr }}\left(\mu \mathrm{A} / \mathrm{cm}^{2}\right)$ & 27 & 37.7 & 26.2 & 25.7 & 23.2 & 18.7 \\
& $\mathrm{~b}_{\mathrm{a}}(\mathrm{mV} / \mathrm{dec})$ & 168 & 214 & 134 & 159 & 163 & 178 \\
& $-\mathrm{b}_{\mathrm{c}}(\mathrm{mV} / \mathrm{dec})$ & 309 & 283 & 370 & 340 & 321 & 372 \\
& $\eta_{\text {pol } \%}$ & - & -39.62 & 2.96 & 4.81 & 14.07 & 30.74 \\
\hline \multirow{5}{*}{ Hp3ylm } & $-\mathrm{E}_{\text {corr }}(\mathrm{mV} / \mathrm{SCE})$ & 240 & 313 & 191 & 160 & 145 & 125 \\
& $\mathrm{I}_{\text {corr }}\left(\mu \mathrm{A} / \mathrm{cm}^{2}\right)$ & 27 & 18.4 & 14.8 & 8.2 & 4.8 & 2.6 \\
& $\mathrm{~b}_{\mathrm{a}}(\mathrm{mV} / \mathrm{dec})$ & 168 & 173 & 143 & 100 & 94 & 69 \\
& $-\mathrm{b}_{\mathrm{c}}(\mathrm{mV} / \mathrm{dec})$ & 309 & 399 & 299 & 198 & 196 & 182 \\
& $\eta_{\text {pol } \%}$ & - & 31.85 & 45.18 & 69.62 & 82.03 & 90.18 \\
\hline
\end{tabular}

\subsection{Surface Morphological Studies}

For studying the surface modifications, scanning electron micrographs of the metal surfaces were taken in the absence and presence of the oximes. A clear difference was noticed between the micrographs of bare copper surface, metal treated with $\mathrm{HNO}_{3}$ and metal treated with $\mathrm{HNO}_{3}$ in the presence of oximes. The textures of Fig. 6(a) and $\mathbf{6 ( b )}$ are entirely different. The smoothness of the bare metal surface was completely lost in the presence of the corrodent nitric acid Fig. 6(b). Surface images of copper specimens in the presence of the heterocyclic oximes Fig. 6(c) and Fig. 6(d) are entirely different from the morphology of metal in nitric acid. This is due the development of a thin passivation layer of oximes on copper surface. On comparing Fig. 6(c) and Fig. 6(d), it may be assumed that a visible thin protective layer of Hp3ylm is adhered on the copper surface which can be regarded as reason for the elevated inhibition efficiency of Hp3ylm than Hp2ylm.

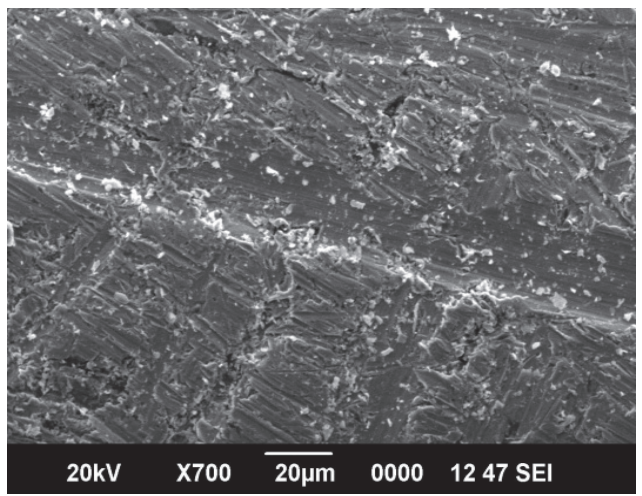

(a)

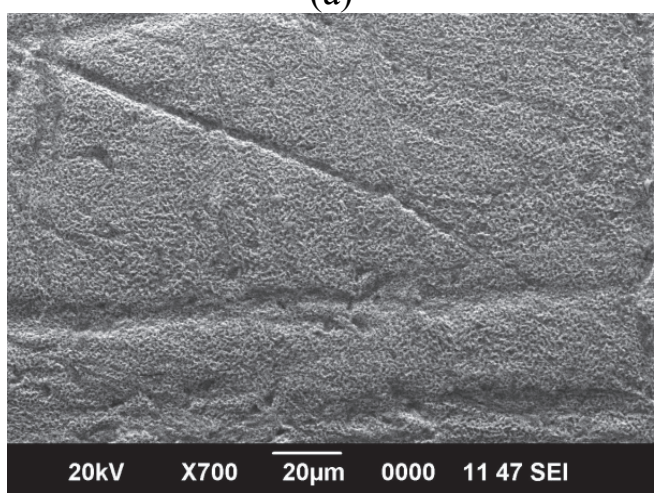

(c)

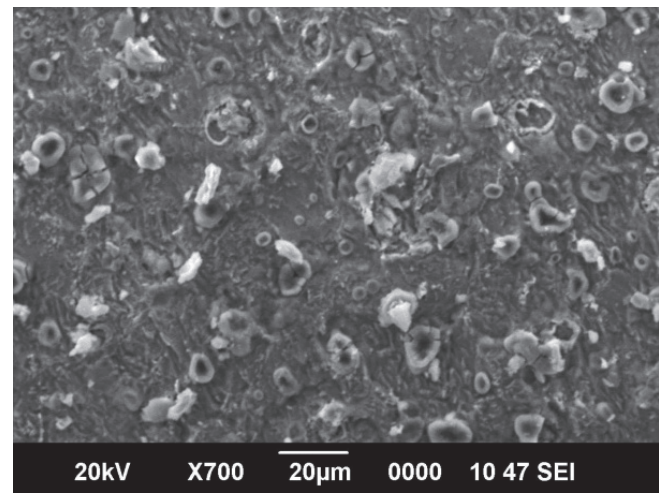

(b)

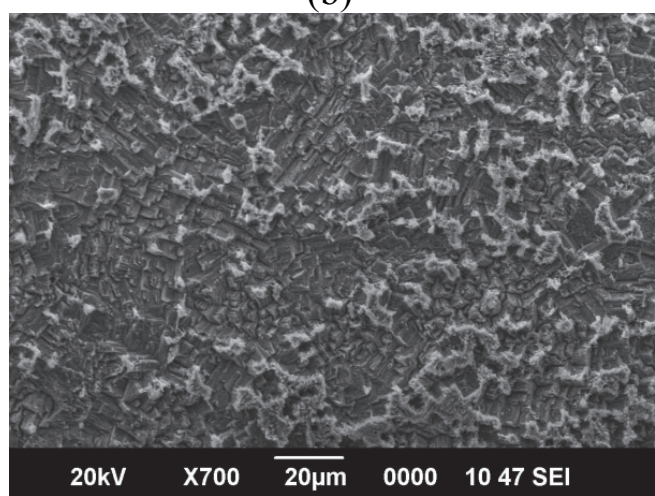

(d)

Fig. 6. SEM images of a) bare metal, b) $\mathrm{Cu}$ in nitric acid $(10 \mathrm{~h})$, c) $\mathrm{Cu}$ with $\mathrm{HNO}_{3}$ and $\mathrm{Hp} 3 y \mathrm{~lm}$ $(1 \mathrm{mM}), \mathrm{d}) \mathrm{Cu}$ with $\mathrm{HNO}_{3}$ and $\mathrm{Hp} 2 \mathrm{ylm}(1 \mathrm{mM})$ 


\subsection{Quantum Chemical Calculations}

Energy of frontier molecular orbitals can be correlated with the corrosion inhibition behaviour of molecules. Interaction of organic molecules on the surface of a metal can be explained by HSAB concept. According to this concept, electron rich molecular orbitals of organic molecules donate electrons to the vacant orbitals of copper atoms on the surface. Greater the energy differences between $\mathrm{E}_{\mathrm{HOMO}}$ and $\mathrm{E}_{\mathrm{LUMO}}$ lesser will be the transference of electron and hence lesser will be the metal-molecule interaction. ${ }^{25,26}$ Quantum chemical calculations were performed by DFT method using GAMESS software. In DFT calculations Beck's three parameter exchange functional and Lee-Yang-Parr correlation functional (B3LYP) was used. ${ }^{27}$ Quantum chemical parameters of Hp2ylm and Hp3ylm like $\mathrm{E}_{\mathrm{HOMO}}, \mathrm{E}_{\mathrm{LUMO}}$ and $\Delta \mathrm{E}$ and HSAB parameters like electronegativity $(\chi)$ and chemical hardness $(\eta)$ of the molecules determined ${ }^{28}$ by equations 8 and 9 are provided in Table 4 . The HOMO and LUMO of the molecules are given in Fig. 7.

$$
\begin{aligned}
& \chi \approx-1 / 2\left(\mathrm{E}_{\mathrm{HOMO}}+\mathrm{E}_{\mathrm{LUMO}}\right) \\
& \eta \approx 1 / 2\left(\mathrm{E}_{\mathrm{HOMO}}-\mathrm{E}_{\mathrm{LUMO}}\right)
\end{aligned}
$$

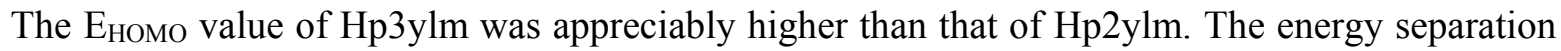
$\Delta \mathrm{E}$ was considerably lower for Hp3ylm than Hp2ylm, which indicate that Hp3ylm has a better corrosion inhibition capacity than Hp2ylm. This also suggests that the energy required to transfer electrons from the HOMO of Hp3ylm to the vacant d-orbitals of copper atoms is lower than that of $\mathrm{Hp} 2 \mathrm{ylm}$. It is possible to determine the approximate number of electrons transferred $(\Delta \mathrm{N})$ from donor to acceptor molecules quantum chemically by equation 10 . For this calculation, chemical hardness of $\mathrm{Cu}$ metal is assumed as zero and the approximate electronegativity of bulk $\mathrm{Cu}$ is taken as $4.48 \mathrm{eV}{ }^{29}$

$$
\Delta \mathrm{N}=\frac{\chi_{C u-} \chi_{\text {inhib }}}{2\left(\eta_{C u+} \eta_{\text {inhib })}\right.}
$$

It is evident from Table 4 that the number of electrons transferred from the HOMO to the vacant orbital of copper atom is higher for Hp3ylm than Hp2ylm. This is a clear indication of the stronger interaction of Hp3ylm on Cu surface than Hp2ylm.

Table 4. Quantum chemical parameters of Hp2ylm and Hp3ylm on copper

\begin{tabular}{lcccccr}
\hline \multicolumn{1}{c}{ Molecule } & Eномо $(\mathbf{e V})$ & ELumo $(\mathbf{e V})$ & $\Delta \mathbf{E}(\mathbf{e V})$ & $\mathbf{X}$ & $\boldsymbol{\eta}$ & $\Delta \mathbf{N}$ \\
\hline Hp2ylm & -4.563 & 0.879 & 5.442 & 1.842 & 2.721 & 0.9478 \\
Hp3ylm & -4.452 & 0.838 & 5.290 & 1.807 & 2.645 & 0.9816 \\
\hline
\end{tabular}

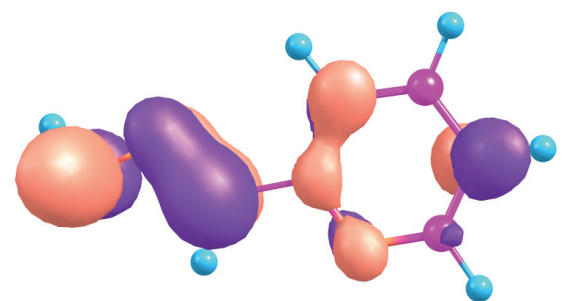

Hp2ylm (HOMO)

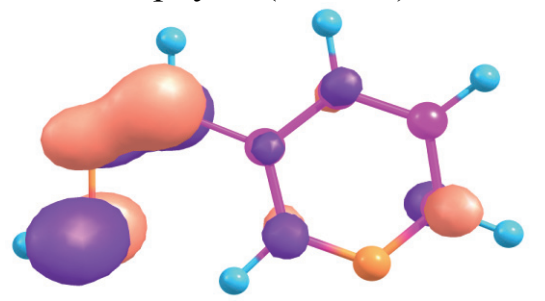

Hp3ylm (HOMO)

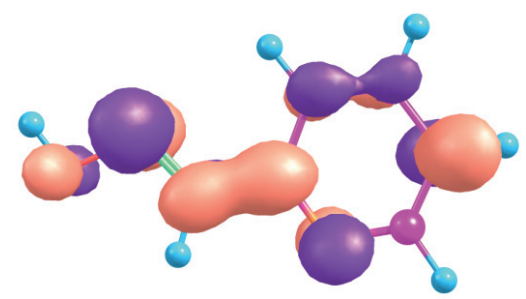

Hp2ylm (LUMO)

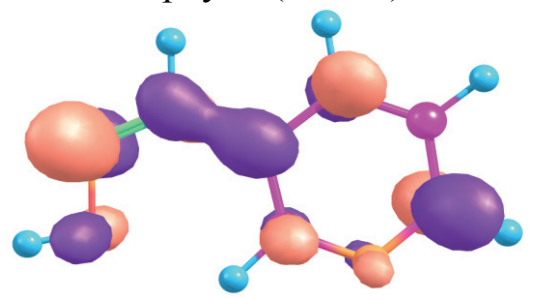

Hp3ylm (LUMO)

Fig. 7. HOMO and LUMO of Hp2ylm and Hp3ylm 


\section{Conclusions}

On comparing the inhibition efficiency of two heterocyclic derivatives on copper in $0.1 \mathrm{M} \mathrm{HNO}_{3}$, Hp3ylm exhibited very high corrosion inhibition efficiency on copper metal than Hp2ylm in $0.1 \mathrm{M}$ $\mathrm{HNO}_{3}$ as per electrochemical studies. At $1 \mathrm{mM}$ concentration, Hp2ylm and Hp3ylm displayed 26.93\% and $90.14 \%$ of corrosion inhibition efficiency respectively according to impedance studies. The heterocyclic oxime Hp3ylm obeyed Langmuir adsorption isotherm on copper metal in nitric acid. Adsorption studies revealed that both physisorption and chemisorption involved in the interaction of Hp3ylm on the copper metal surface. Polarization studies revealed that both oximes act as a mixed type corrosion inhibitor. The reason for difference in the corrosion protection capacity of molecules was explained by quantum chemical investigations.

\section{Acknowledgement}

Authors are grateful to UGC for providing the financial assistance for the research work.

\section{Experimental}

\subsection{Synthesis and Characterisation}

Chemicals used for the study such as pyridine-2-aldehyde, pyridine-3-aldehyde, hydroxyl amine hydrochloride, sodium acetate and nitric acid were of analytical grade (Merck Millipore). Carbon, hydrogen, and nitrogen content of the oximes were determined by microanalysis using Vario EL III model CHN analyzer (Elementar make). Electronic spectrum was measured in DMSO medium using a Shimadzu make spectrophotometer. F-IR spectra of the oximes were recorded using $\mathrm{KBr}$ disc technique in the range $4000-400 \mathrm{~cm}^{-1}$ on a Shimadzu FT-IR Spectrometer. NMR spectra $\left({ }^{1} \mathrm{H}: 300\right.$ and ${ }^{13} \mathrm{C}: 75 \mathrm{MHz}$ ) of the compounds were taken in DMSO- $\mathrm{d}_{6}$ on a Bruker AMX-400 NMR spectrometer.

Heterocyclic oximes Hp2ylm and Hp3ylm ${ }^{30}$ were prepared by the following method. To a 1:1 ratio mixtures of hydroxylamine hydrochloride and sodium acetate in aqueous medium, alcoholic solution of pyridine-2-carbaldehyde/pyridine-3-carbaldehyde was added with constant stirring, refluxed for 20 minutes and cooled. The separated oxime was filtered, washed with alcohol- water mixture $(1: 1)$ and dried.

Hp2ylm CHN calcd. for $\mathrm{C}_{6} \mathrm{H}_{6} \mathrm{~N}_{2} \mathrm{O}: \mathrm{C}, 59.01 ; \mathrm{H}, 4.91 ; \mathrm{N}, 22.95 ; \mathrm{O}, 13.12 \%$. Found. C, 58.45; $\mathrm{H}$, $4.54 ; \mathrm{N}, 22.56 ; \mathrm{O}, 12.72 \%$; IR $(\mathrm{KBr}): v_{\mathrm{C}=\mathrm{N}} 1593 \mathrm{~cm}^{-1} .{ }^{1} \mathrm{HNMR}: \delta_{\mathrm{OH}} 9.65, \delta_{\mathrm{CH}=\mathrm{N}} 8.27 .{ }^{13} \mathrm{Cnmr}: \delta_{\mathrm{CH}=\mathrm{N}}$ 136.82. Mass: $\mathrm{M}^{+}$peak (base peak) $\mathrm{m} / \mathrm{z}: 122 . \mathrm{Mp}=135{ }^{\circ} \mathrm{C}$; soluble in $\mathrm{H}_{2} \mathrm{O}$, ethanol, acidic medium, pale pink colour, $\pi \rightarrow \pi^{*}$ and $\mathrm{n} \rightarrow \pi^{*}$ electronic transitions at $39062 \mathrm{~cm}^{-1}$ and $29411 \mathrm{~cm}^{-1}$.

Hp3ylm CHN calcd for $\mathrm{C}_{6} \mathrm{H}_{6} \mathrm{~N}_{2} \mathrm{O}: \mathrm{C}, 59.01 ; \mathrm{H}, 4.91 ; \mathrm{N}, 22.95 ; \mathrm{O}, 13.12 \%$. Found. C, 58.29; H, 4.44; N, 22.47 ; O, 12.97\%; IR (KBr): $v_{\mathrm{C}=\mathrm{N}} 1633 \mathrm{~cm}^{-1} .{ }^{1} \mathrm{HNMR}: \delta_{\mathrm{OH}} 8.66, \delta_{\mathrm{CH}=\mathrm{N}} 8.17 .{ }^{13} \mathrm{Cnmr}: \delta_{\mathrm{CH}=\mathrm{N}}$ 133.74. Mass: $\mathrm{M}^{+}$peak and base peak $\mathrm{m} / \mathrm{z}: 122 . \mathrm{Mp}=108{ }^{0} \mathrm{C}$; white colour, soluble in $\mathrm{H}_{2} \mathrm{O}$, ethanol, acidic medium, $\pi \rightarrow \pi^{*}$ and $\mathrm{n} \rightarrow \pi^{*}$ electronic transitions $38986 \mathrm{~cm}^{-1}$ and $29411 \mathrm{~cm}^{-1}$.

Molecular structures of the heterocyclic oximes Hp2ylm and Hp3ylm are given in Fig. 8 and Fig. 9.

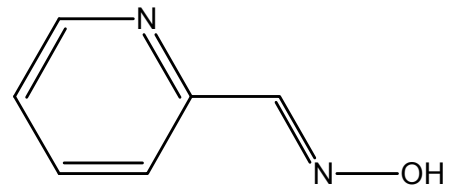

Fig. 8. Structure of Hp2ylm

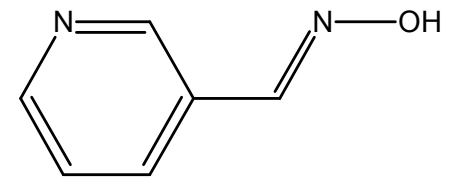

Fig. 9. Structure of Hp3ylm 


\subsection{Electrochemical Measurements}

Approximate composition of copper metal was determined by EDS method was $99.5 \% \mathrm{Cu}$, $0.0033 \% \mathrm{Ni}, 0.0079 \% \mathrm{Mn}, 0.0089 \% \mathrm{Al}$, and $0.13 \% \mathrm{Si}$. Copper strips used for the investigation was abraded by various grades of emery sheets $(200,1000,1500$, and 2000). The metal surface was degreased with acetone, washed with water and dried. Using $0.1 \mathrm{M} \mathrm{HNO}_{3}$, solutions of $\mathrm{Hp} 3 y \mathrm{ym}$ and Hp2ylm were prepared in the range 0.2-1.0 mM.

Electrolytic cell used for the study consisted of three electrodes namely saturated calomel electrode (reference), platinum electrode (inert, area $1 \mathrm{~cm}^{2}$ ) and copper strip (working electrode- area $1 \mathrm{~cm}^{2}$ ). To attain open circuit potential, copper electrode was exposed to the aggressive solution for $1 \mathrm{~h}$. For eliminating the polarization effects, copper specimen and SCE was kept very close. Computer controlled electrochemical workstation (Ivium compactstat-e model) was used to conduct electrochemical analysis.

\subsection{Impedance Spectroscopic Studies}

EIS studies were carried out at OCP over $1 \mathrm{KHz}-100 \mathrm{mHz}$ frequency range with a potential perturbation amplitude of $10 \mathrm{mV}$. After the experiment, Nyquist plots were analyzed using equivalent circuit. From the charge resistance values of copper specimens, percentage of corrosion inhibition efficiency was calculated using equation $11 .{ }^{31,32}$

$$
\eta_{\mathrm{EIS}} \%=\frac{\mathrm{R}_{\mathrm{ct}}-\mathrm{R}_{\mathrm{ct}}^{\prime}}{\mathrm{R}_{\mathrm{ct}}} \times 100,
$$

where $R_{c t}$ and $R_{\text {ct }}$ are the charge transfer resistances of copper electrode in the presence and absence of oximes respectively.

\subsection{Tafel Polarization Studies}

Copper electrodes were scanned between -250 to $+250 \mathrm{mV}$ Vs corrosion potential ( $\mathrm{E}_{\text {corr }}$ ). Throughout the experiment scanning rate of $1 \mathrm{mV} / \mathrm{sec}$ was used. By analysing the Tafel polarization curves, corrosion current densities $\left(\mathrm{I}_{\text {corr }}\right)$ and anodic and cathodic slopes were obtained. Corrosion protection efficiencies $\left(\eta_{\text {pol }} \%\right)$ of oximes on copper in $0.1 \mathrm{M} \mathrm{HNO}_{3}$ was determined ${ }^{33,34}$ using Eq. (12).

$$
\eta_{\text {pol }} \%=\frac{I_{\text {corr }}-I_{\text {corr }}^{\prime}}{I_{\text {corr }}} \times 100
$$

In the above equation, $\mathrm{I}_{\text {corr }}$ and I' corr represent the corrosion current densities of copper in the absence and presence of oxime.

\subsection{Quantum chemical studies}

To correlate the electronic structure and corrosion protection efficacy of oximes quantum chemical studies were performed using DFT method using GAMESS software.

\subsection{Surface morphological investigations}

To investigate the surface modifications on the copper metal specimens during the process of corrosion and corrosion inhibition, scanning electron microscopic studies were conducted using Joel JSM-6390 LV/JED-2300 model microscope. 


\section{Acknowledgement}

The authors would like to thank the anonymous referees for constructive comments on earlier version of this paper.

\section{References}

1. Khaled K. F., Amin M. A., and Al-Mobarak N. A. (2010) On the corrosion inhibition and adsorption behaviour of some benzotriazole derivatives during copper corrosion in nitric acid solutions: a combined experimental and theoretical study. J. Appl. Electrochem., 40(3), 601613.

2. Raphael V. P., Thomas K. J., Shaju K. S., and Paul A. (2014) Corrosion inhibition investigations of 3-acetylpyridine semicarbazone on carbon steel in hydrochloric acid medium. Res. Chem. Intermediat., 40(8), 2689-2701.

3. John S., and Joseph A. (2012) Electro analytical, surface morphological and theoretical studies on the corrosion inhibition behaviour of different 1,2,4-triazole precursors on mild steel in $1 \mathrm{M}$ hydrochloric acid. Mater. Chem. Phys., 133, 1083-1091.

4. Karthik G., and Sundaravadivelu M. (2016) Investigations of the inhibition of copper corrosion in nitric acid solutions by levetiracetam drug," Egyptian Journal of Petroleum, 25(4),481-493

5. Khan P. F., Shanthi V., Babu R. K., Muralidharan M., and Barik R. C. (2015) Effect of benzotriazole on corrosion inhibition of copper under flow conditions. J. Enviorn. Chemi. Eng., 3(1), 10-19.

6. Gopi D., Govindaraju K. M., and Kavitha L. (2010) Investigation of triazole derived Schiff bases as corrosion inhibitors for mild steel in hydrochloric acid medium. J. Appl. Electrochem., 40, 1349-1356.

7. Hosseini S. M. A., and Azimi A. (2009) The inhibition of mild steel corrosion in acidic medium by 1-methyl-3-pyridin-2-yl-thiourea. Corros. Sci., 51(4), 728-732.

8. Liu P., Fang X., Tang Y., Sun C., and Yao C. (2011) Electrochemical and Quantum Chemical Studies of 5-Substituted Tetrazoles as Corrosion Inhibitors for Copper in Aerated $0.5 \mathrm{M} \mathrm{H}_{2} \mathrm{SO}_{4}$ Solution. Mater. Sci. Appl., 2, 1268-1278.

9. Dafali A., Hammouti B., Mokhlisse R., and Kertit S., (2003) Substituted uracils as corrosion inhibitors for copper in 3\% NaCl solution. Corros. Sci., 45(8), 1619-1630.

10. Kuriakose N., Thomas K. J., Raphael V. P., Shaju K. S., (2014) Electrochemical Impedance Spectroscopy and Potentiodynamic Polarization Analysis on Anticorrosive Activity of Thiophene-2-Carbaldehyde Derivative in Acid Medium. Indian J. Mater. Sci., 2014, Article ID 124065, doi:10.1155/2014/124065.

11. Ouali I. E., Hammouti B., Aouniti A., Benabdellah M., and Kertit S., (2011) Electrochemical behaviour of N,S-containing corrosion inhibitor for C38 Steel in molar HCl. Der Pharma Chemica, 3(5), 294-300.

12. Zarrouk A., Dafali A., Hammouti B., Zarrok H., Boukhris S., and Zertoubi M., (2010) Synthesis, Characterization and Comparative Study of Quinoxalines Functionalized Derivatives towards Corrosion of Mild Steel in Hydrochloric Acid Medium. Inter. J. Electrochemi. Sci., 5, 46-55.

13. Abd El Rehim, S. S., Ibrahim, M. A. M., and Khalid, K. F. (2001) The inhibition of 4-(2'-amino5'-methylphenylazo) antipyrine on corrosion of mild steel in $\mathrm{HCl}$ solution. Mater. Chem. Phy., 70(3), 268-273.

14. Raman A., and labine P., (1986) Reviews on Corrosion Inhibitor Science and Technology, NACE, Houston, Tex, USA.

15. Li X., Deng S., and Fu H., (2009) Synergism between red tetrazolium and uracil on the corrosion of cold rolled steel in $\mathrm{H}_{2} \mathrm{SO}_{4}$ solution. Corros. Sci., 51(6), 1344-1355. 
16. Cafferty M. M., and Hackerman N., (1972) Double layer capacitance of tron and corrosion inhibition with polymethylene diamines. J. Electrochim. Soc., 119(2), 146-154.

17. Achouri M. E., Kertit S., Gouttaya H. M., Nciri B., Bensouda Y., Perez L., Infante M. R., and Elkacemi K. (2001) Corrosion inhibition of some Gemini surfactants in the series of alkanediayl-a,x-bis(dimethyltetradecyl ammonium bromide). Prog. Org. Coat., 43, 267-273.

18. El-Shafei A. A., Moussa M. N. H., and El- Far A. A., (2001) The corrosion inhibition character of thiosemicarbazide and its derivatives for C-steel in hydrochloric acid solution. Mater. Chem. Phys., 70(2), 175-180.

19. Shukla S. K., Quraishi M. A., and Prakash R., (2008) A self-doped conducting polymer polyanthranilic acid: for mild steel in acid solution. Corros. Sci., 50, 2867-2872.

20. Fuchs-Godec R., (2007) Inhibitory effect of non -ionic surfactants of the TRITON-X series on the corrosion of carbon steel in sulphuric acid. Electrochim. Acta., 52, 4974-4981.

21. Umoren S. A., Eduok U. M., and Oguzie E. E., (2008) Corrosion inhibition of mild steel in $1 \mathrm{M}$ $\mathrm{H}_{2} \mathrm{SO}_{4}$ by polyvinyl pyrrolidone and synergistic iodide additives. Portugaliae Electrochim. Acta., 26(6), 533-546.

22. Pourbaix M. (1975) Atlas of Elecrochemical Equilibria in Aqueous Solutions. NACE, Houston, TX.

23. Johnson H. E., and Leja J. (1965) On the Potential $/ p \mathrm{H}$ diagrams of the $\mathrm{Cu}-\mathrm{NH}_{3}-\mathrm{H}_{2} \mathrm{O}$ and $\mathrm{Zn}-\mathrm{NH}_{3}-\mathrm{H}_{2} \mathrm{O}$ systems technical notes. J. Electrochem. Soc., 112(6), 638-641.

24. Smyrl W. H., Bockris J., Conway B. E., Yeager E., and White R. E. (1981) Comprehensive Treatise of Electrochemistry. 4, Plenum Press, New York.

25. Mahdavian M., and Ashhari S. (2010) Corrosion inhibition performance of 2mercaptobenzimidazole and 2-mercaptobenzoxazole compounds for protection of mild steel in hydrochloric acid solution. Electrochim. Acta., 55, 1720-1724.

26. Bentiss F., Lebrini M., Lagrenee M., Traisnel M., Elfarouk A., and Vezin H. (2007) The influence of some new 2,5-disubstituted 1,3,4- thiadiazoles on the corrosion behaviour of mild steel in $1 \mathrm{M} \mathrm{HCl}$ solution: AC impedance study and theoretical approach. Electrochim. Acta., 52, 6865-6872.

27. Sastri V. S., and Perumareddi J. R. (1997) Molecular orbital theoretical studies of some organic corrosion inhibitors. Corrosion 53(8) 617-622.

28. Ferreira E. S., Giacomelli C., Giacomelli F. C., and Spinelli A. (2004). Evaluation of the inhibitor effect of L-ascorbic acid on the corrosion of mild steel. Mater. Chem. Phys., 83(1) 129-134.

29. Xia S., Qiu M., Yu L., Liu F., and Zhao H. (2008) Molecular dynamics and density functional theory study on relationship between structure of imidazoline derivatives and inhibition performance. Corros. Sci., 50, 2021-2029.

30. Malpica A., Calzadilla M., Baumrucker J., Jimenez J., Lopez L., Escobar G., and Montes C. (1994) Kinetics and Mechanism for Oxime Formation from Pyridine-2-, -3-, and -4carboxaldehydes. J. Org. Chem., 59 (12), 3398-3401.

31. Fiala A., Chibani A., Darchen A., Boulkamh A., and Djebbar K. (2007) Investigations of the inhibition of copper corrosion in nitric acid solutions by ketene dithioacetal derivatives. Appl. Sur. Sci., 253(24), 9347-9356.

32. Khaled K.F. (2010) Corrosion control of copper in nitric acid solutions using some amino acids - A combined experimental and theoretical study. Corros. Sci., 52(10), 3225-3234.

33. Hanza P., Naderi A.R., Kowsari E., and Sayebani M. (2015) Corrosion behavior of mild steel in $\mathrm{H}_{2} \mathrm{SO}_{4}$ solution with 1,4 -di[1'-methylene-3-' methyl imidazolium bromide]- benzene as an ionic liquid, Corros. Sci., 107, 96-106.

34. Soltani N., Behpour M., Ghoreishi S. M., and Naeimi H. (2010) Corrosion inhibition of mild steel in hydrochloric acid solution by some double Schiff bases. Corros. Sci., 52(4), 1351-1361. 
(C) 2018 by the authors; licensee Growing Science, Canada. This is an open access article distributed under the terms and conditions of the Creative Commons Attribution (CC-BY) license (http://creativecommons.org/licenses/by/4.0/). 